\title{
Nurses' Perceptions of Barriers and Facilitators to Implement EBP in the Maldives
}

\author{
Fathimath Shifaza, David Evans, and Helen Bradley \\ School of Nursing and Midwifery, University of South Australia, GPO Box 2471, Adelaide, SA 5001, Australia \\ Correspondence should be addressed to Fathimath Shifaza; shify003@mymail.unisa.edu.au
}

Received 30 July 2014; Accepted 19 October 2014; Published 9 November 2014

Academic Editor: Jenny M. Wilkinson

Copyright (C) 2014 Fathimath Shifaza et al. This is an open access article distributed under the Creative Commons Attribution License, which permits unrestricted use, distribution, and reproduction in any medium, provided the original work is properly cited.

\begin{abstract}
This study aims to explore the barriers to evidence based practice (EBP) experienced by nurses working in a Maldivian healthcare organisation. A total of 400 questionnaires were distributed to nurses in 5 healthcare facilities and 198 completed questionnaires were returned. The results of this study show that the perceived barriers by the nurses in the Maldives are not significantly different from those reported in developed countries. For healthcare organisations in the Maldives, it is necessary to have a clear understanding of the barriers and facilitators to research practice in order to implement EBP. The study showed that the major barriers to research use were that "the relevant literature is not compiled in one place," there is "insufficient time on the job to implement new ideas," and "administration will not allow implementation." The key facilitating factors for EBP include support, encouragement, and recognition by the management and administration. The findings of this study can be useful for determining strategies that can be introduced in the clinical setting to use EBP. Healthcare organisations must continue their support in order to decrease the barriers and optimise care in healthcare facilities.
\end{abstract}

\section{Introduction}

EBP has become the predominant model of care that has gained recognition for facilitating the transfer of research evidence into clinical practice. Using EBP means integrating the best available information with clinical expertise and patient values to achieve optimal health outcomes [1]. As a consequence, it is a critical way to strengthen the practice of nursing.

The government of the Maldives is concerned with improving the health and wellbeing of the people of Maldives and thus has articulated a firm commitment to promoting EBP. However, EBP is a new paradigm in the Maldives and nursing research is still at an early stage of development. While it is well recognised that the Maldives needs to facilitate an EBP and research culture, political, economic, geographical, and social factors hinder the use of research evidence. Therefore, this study was initiated to identify the barriers and facilitators for EBP in the Maldives.

\section{Barriers and Facilitators to Research Use}

Barriers to the use of research in clinical practice have been cited frequently. Studies have investigated possible barriers to the adoption of EBP by nurses [2-11]. These studies have identified common barriers across a number of different countries. Historically, the primary barriers to the use of research in practice have been linked to the support provided by the organisation in which nurses work, the nurses' research values and skills, the quality of research, and how the research is communicated or presented [12]. A meta-analysis conducted by Ashley [2] of barrier and facilitators to research utilisation concluded that the top barriers were insufficient time, lack of understanding of statistics, lack of authority to change patient care, and lack of time to read literature.

Numerous facilitators of research use have been identified in the literature. These facilitators include managerial and peer support, availability of time to review and implement research findings, availability of relevant research, colleagues' 
support, supportive policies, and training opportunities [11, $13,14]$. Barriers and facilitators to research utilization were categorized into organizational factors, individual factors, communicational factors, and quality of research.

2.1. Organisational Factors. Organisational factors are barriers and limitations perceived in the setting where the research will be used $[6,12]$. Time constraints are a serious barrier in research utilisation $[9,11-13]$. Time to read, evaluate, analyse, disseminate, and implement research evidence is another barrier cited by nurses in many countries. In a survey undertaken in Ireland by Parahoo [9], nurses identified that seven of the top 10 perceived barriers to implementing research findings were related to organisational factors. The top barrier was insufficient authority to instigate change in the practice setting. This factor was also identified by Hutchinson and Johnston [13] in Australia and by nurses in Hong Kong [11]. Inadequate facilities [3, 11, 15] and lack of support for implementing research $[5,13]$ have also been cited as major organisational barriers.

2.2. Individual Factors. Commonly identified individual barriers reported in the literature include lack of knowledge about research methods, poor awareness of research findings, and negative attitude towards research $[3,6]$. A study conducted in Spain found that the top three barriers were related to the individual characteristics of the nurses, particularly their beliefs about the value of research and confidence in their research skills [8]. Being incapable of evaluating research quality was rated the greatest barrier by MorenoCasbas et al. [8]. Inadequate skills in critical appraisal, lack of skills in accessing research and evaluating its quality, lack of confidence to implement change, and unfamiliarity with the research language have also been reported as barriers in the literature $[12,15]$.

2.3. Communicational Factors. Communication or how the research is presented is another major barrier highlighted in the literature. The physical accessibility of published research [16], literature not compiled in one location $[3,15]$, lack of awareness of available research literature [15], and statistical analyses not being understandable $[9,17]$ have all been reported frequently in the literature.

2.4. Quality of Research. An equally important factor may be the quality of published research. Methodological inadequacies, poor justification of research conclusions, and conflicting results are all problematic for the novice researcher. Uncertainty of research results and articles not published fast enough are also components of the quality of research and the characteristics of innovation [6].

This review of the literature suggests that identifying the barriers of research use and strategies that facilitate organisational support in healthcare facilities will allow for improved program planning and the removal of perceived barriers. This organisational support will facilitate EBP and ultimately improve the quality of care provided by healthcare facilities. However, this review highlighted that factors related to the individual practitioner, communication, and quality can also influence the implementation of research into practice.

\section{Purpose of the Study}

The purpose of this study was to explore and describe the barriers and facilitators experienced by nurses, as well as nurses' attitudes and awareness of EBP within the health service corporation in the Maldives. The research question was as follows.

What are the perceived barriers and facilitators to research use among nurses in the Maldives?

\section{Study Design}

The study sought to identify the barriers and facilitators of research use in the healthcare facilities of Maldives. It employed a survey design and used the BARRIER Scale. It was the first phase of a larger action research project designed to promote EBP in the healthcare facility.

4.1. Setting and Participants. The study was undertaken within the five healthcare service centres of Male Health Service Corporation (MHSC), the Maldives. The MHSC provides services at a territory hospital (IGMH) in the capital city of Malé, one community hospital (Hulhumalé Hospital), and two multipurpose health centres (Villigilli Health Centre and Malé Health Centre). In addition, it includes a specialty clinic (Thalassemia Clinic). There are approximately 1,600 employees, one-third of whom are nurses. All RNs, midwifes, and ENs were invited to participate in the study. The survey was undertaken from February to June 2012.

4.2. Sample. A convenience sampling strategy was employed to distribute 400 questionnaires to all nurses working during the survey period. The purpose of the study was explained to all participants in a cover letter included with the questionnaire. The term nurses were operationally defined as all grades of nurses and midwives working in any area of the healthcare service centres. Questionnaires were distributed by the researcher to the ward managers in each of the healthcare service centres for distribution to the nurses. Participants were asked to return the completed questionnaires within two weeks.

4.3. Instrument. The BARRIER Scale [6] was used to explore the perceived barriers to research use and identify the variables that were related to research use in the Maldives. Additional questions were used to collect demographics such as age, educational level, years of experience, and work place. Permissions were obtained from the developers of the BARRIER Scale.

The BARRIER Scale consists of 29 items and has been validated in various settings to assess barriers for research utilisation and implementation of EBP. Funk et al. [6] employed factor analysis in order to establish the reliability of the instrument. Funk et al. clustered these barriers around four factors that are congruent with dimensions in Rogers's 
[18] diffusion of innovation theory. These four factors were labelled

(i) characteristics of the adopter ( $\mathrm{N}$, nurse) (eight items),

(ii) characteristics of the organisation ( $\mathrm{S}$, setting barriers and limitations) (eight items),

(iii) characteristics of the innovation ( $R$, quality of the research) (six items),

(iv) characteristics of the communication ( $\mathrm{P}$, presentation and accessibility of the research) (six items).

The characteristics of the adopter measured the nurses' values, skills, and awareness of research. The characteristics of the organisation measured the nurses' perception about limitations and barriers in the work setting. The characteristics of the innovation measured nurses' perceptions of the quality of research. The characteristics of the communication measured nurses' perceptions about the presentation and accessibility of the research. The internal consistency reliability of the tool was tested using Cronbach's alpha coefficient. Cronbach's alpha coefficients for the four factors on the BARRIER Scale were found to be 0.65 to 0.80 [6]. Cronbach's alpha is an index of the degree to which all of the different items in a scale measure the same attribute [19].

The instrument employs a four-point scale $(1=$ no extent, $2=$ to a little extent, $3=$ moderate extent, and $4=$ to a great extent). In addition, a "no opinion" option was also offered; however, the "no opinion" responses were not included in the scoring. The respondents were asked to rate to what extent they perceived each item as a barrier to using research findings. The questionnaire also offered space for the respondents to add any perceived barriers not mentioned in the questionnaire and to rank the three greatest barriers. Further, the nurses were asked to suggest factors that might facilitate research utilisation.

4.4. Data Analysis. Quantitative data were coded and analysed using SPSS 20 software. The normality of the data was obtained through descriptive statistics of means, standard deviations, and frequencies. Analysis was performed as follows. Items 1 to 29 on the BARRIER Scale were loaded into one of the four factors identified by Funk et al. [6]. For each subscale, the mean scores were added and then the sum was divided by the number of items in the subscale in order to attain a comparable figure. Items for which individuals responded "no opinion" or left blank were eliminated. Therefore, the divisor for the mean for each factor was the number of items with valid responses. In order to identify the single largest barriers to research utilisation, the number of respondents who reported each barrier as a moderate or great barrier was calculated, and items were ranked ordered accordingly. Descriptive analysis was used for items 30 to 33-other barriers to research utilisation-to identify unique barriers to research utilisation as perceived by the nurses in the Maldives. In this study, item 34-the three greatest barriers to nurses' use of research-was defined as those with the greatest mean score for the entire sample. Content analysis was used for the open-ended question in item 35-facilitators to research utilisation.
TABLE 1: Demographics.

\begin{tabular}{lcc}
\hline Demographics & Number & $\%$ \\
\hline Age & & \\
Up to 29 years & 94 & 47.2 \\
30-39 years & 85 & 42.7 \\
40-49 years & 14 & 7.0 \\
50-59 years & 1 & 0.5 \\
Gender & & \\
Male & 25 & 12.6 \\
Female & 173 & 87.4 \\
Qualification & & \\
Bachelor's degree & 31 & 15.7 \\
Diploma nursing/midwifery & 127 & 64.1 \\
Certificate level & 38 & 19.2 \\
Missing values & 2 & 1 \\
Setting & & \\
IGMH & 161 & 81.3 \\
Hulhumalé Hospital & 23 & 11.6 \\
Villigili Health Centre & 2 & 1.0 \\
National Thalassemia Centre & 11 & 5.6 \\
Malé Health Centre & - & - \\
Position & & \\
Ward managers & 11 & 5.6 \\
Deputy managers & 18 & 9.1 \\
Senior RNs & 3 & 1.5 \\
RNs/midwives & & \\
ENs & & \\
Years of experience & & \\
in the Maldives & & \\
overseas & & \\
\hline & & \\
& &
\end{tabular}

\section{Ethics}

Participation in this study was voluntary and the anonymity of the participants was preserved because no identifying data were collected. Ethical approvals were obtained from the University of South Australia Human Research Committee (HREC) and the Maldives Nation Health Research Committee (NHRC) prior to commencing the study.

\section{Results}

A total of 198 nurses participated in this survey, representing a $49.5 \%$ response rate. The majority $(n=173,87.4 \%)$ of the respondents were female and the mean age was 30.5 years (range 19 to 52 years). The majority of participants were RNs $(n=127,64.1 \%)$, followed by ENs $(n=38,19.3 \%)$. On average, they had been employed in the nursing profession for 6.8 years (range 15 days to 28 years). Of the 198 respondents, $n$ $=60(30 \%)$ had overseas work experience ranging from one to 12 years. The greatest response to the questionnaire came from IGMH (see Table 1).

To understand how frequently nurses search for information in their clinical practice, participants were asked how often they seek out research evidence. The results show that $46.1 \%$ search occasionally, $16.6 \%$ search often, and $5.2 \%$ search very often for evidence and research information. It 
TABLE 2: BARRIER Scale items.

\begin{tabular}{|c|c|c|c|c|}
\hline BARRIER Scale items & Mean & $\begin{array}{l}\text { Standard } \\
\text { deviation }\end{array}$ & $\begin{array}{c}\text { Moderate or } \\
\text { great barriers } \\
(\%)\end{array}$ & $\begin{array}{c}\text { No opinion or } \\
\text { nonresponse } \\
(\%)\end{array}$ \\
\hline \multicolumn{5}{|l|}{ Organisational factors } \\
\hline There is insufficient time on the job to implement new ideas & 3.26 & 0.88 & 70.7 & 4.5 \\
\hline $\begin{array}{l}\text { The nurse does not feel she/he has enough authority to change patient care } \\
\text { procedures }\end{array}$ & 3.11 & 1.04 & 70.2 & 1.5 \\
\hline Administration will not allow implementation & 3.17 & 0.98 & 69.2 & 4.0 \\
\hline Physicians will not cooperate with implementation & 3.17 & 0.92 & 68.7 & 3.0 \\
\hline The nurses does not have time to read research & 3.12 & 1.03 & 65.2 & 2.5 \\
\hline The facilities are inadequate for implementation & 3.01 & 1.15 & 61.6 & 3.0 \\
\hline Other staff are not supportive of implementation & 2.94 & 0.95 & 61.6 & 5.1 \\
\hline The nurse feels results are not generalisable to own setting & 2.46 & 1.22 & 56.1 & 1.5 \\
\hline \multicolumn{5}{|l|}{ Individual factors } \\
\hline The nurse does not feel capable of evaluating the quality of the research & 3.16 & 1.01 & 67.7 & 4.5 \\
\hline The nurse sees little benefit for self & 2.50 & 0.97 & 66.2 & 3.0 \\
\hline The nurse is unwilling to change/try new ideas & 2.34 & 0.95 & 60.6 & 3.5 \\
\hline There is no documented need to change practice & 2.62 & 0.84 & 56.1 & 4.0 \\
\hline The nurse feels the benefit of changing practice will be minimal & 2.53 & 1.00 & 54.0 & 14.6 \\
\hline $\begin{array}{l}\text { The nurse is isolated from knowledgeable colleagues with whom to discuss the } \\
\text { research }\end{array}$ & 2.95 & 0.94 & 52.5 & 25.8 \\
\hline The nurse is unaware of the research & 2.64 & 0.98 & 38.4 & 15.7 \\
\hline The nurse does not see the value of research for practice & 1.62 & 0.81 & 18.2 & 14.1 \\
\hline \multicolumn{5}{|l|}{ Communicational factors } \\
\hline Statistical analysis is not understandable & 2.85 & 0.35 & 83.3 & 2.5 \\
\hline The relevant literature is not compiled in one place & 3.32 & 0.89 & 80.3 & \\
\hline Research reports/articles are not readily available & 2.98 & 0.94 & 56.6 & 15.7 \\
\hline The research is not relevant to the nurse's practice & 1.72 & 1.00 & 24.7 & 14.6 \\
\hline Implications for practice are not made clear & 1.70 & 0.95 & 21.7 & 16.2 \\
\hline The research is not reported clearly and readably & 1.61 & 0.90 & 19.2 & 25.8 \\
\hline \multicolumn{5}{|l|}{ Quality of research } \\
\hline The research has methodological inadequacies & 2.04 & 1.04 & 48.5 & 1.0 \\
\hline Research reports/articles are not published fast enough & 2.05 & 1.05 & 45.5 & 3.5 \\
\hline The conclusions drawn from the research are not justified & 2.07 & 1.03 & 44.9 & 4.0 \\
\hline The nurse is uncertain whether to believe the results of the research & 2.22 & 1.10 & 43.4 & 8.6 \\
\hline The research has not been replicated & 1.98 & 1.21 & 33.8 & 14.1 \\
\hline The literature reports conflicting results & 1.88 & 1.01 & 37.9 & 10.1 \\
\hline \multicolumn{5}{|l|}{ Additional item } \\
\hline The amount of research information is overwhelming & 2.59 & 0.83 & 41.9 & 5.1 \\
\hline
\end{tabular}

was also noted that $32.1 \%$ never or hardly ever search for research evidence for their clinical practice.

The research participants were also asked how they usually sought the required information: asking colleagues, reading journals, searching bibliographic databases, searching the internet, attending professional development course, or asking the librarian. The majority $(n=111,56.6 \%)$ of nurses responded that they undertook a general internet search, while $26.6 \%$ asked their colleagues, $8.7 \%$ read journals, and only $5.1 \%$ searched bibliographic databases in order to attain the information they needed. Although EBP has been widely discussed in the literature, 55\% nurses were slightly unfamiliar or not familiar with the term EBP, and $45 \%$ only had a moderate understanding of the term.

6.1. BARRIER Scale. For the organisational factors, the biggest barriers reported were "insufficient time on the job to implement new ideas" (70.7\%), "nurse does not feel she/he has enough authority to change patient care procedures" 
TABLE 3: Facilitators to research use.

\begin{tabular}{|c|c|c|}
\hline Theme & Frequency & Illustrative responses \\
\hline Time & 45 & $\begin{array}{l}\text { (i) Increasing availability of time } \\
\text { (ii) Time to use research } \\
\text { (iii) Allocating time period for research work } \\
\text { (iv) Providing study leave/conference leave } \\
\text { (v) Increasing time available for reviewing and implementing EBP }\end{array}$ \\
\hline Organisational support & 76 & $\begin{array}{l}\text { (i) Good communication is required between the senior management and clinicians } \\
\text { (ii) Acknowledgement of the work } \\
\text { (iii) Encouragement from senior management and other healthcare professionals } \\
\text { (iv) "We need support and encouragement from the management" } \\
\text { (v) Support from hospital management with regard to nurses conducting research projects } \\
\text { (vi) Recognition and rewards } \\
\text { (vii) Opportunity to enhance career } \\
\text { (viii) Enhancing managerial support }\end{array}$ \\
\hline Knowledge & 22 & $\begin{array}{l}\text { (i) Providing training regarding research } \\
\text { (ii) Establishing research clubs } \\
\text { (iii) "At this stage nothing new is learned in the in-service classes-it needs to be challenging" } \\
\text { (iv) Training nurses about research } \\
\text { (v) Having the skills for searching literature } \\
\text { (vi) Advance nurses knowledge }\end{array}$ \\
\hline Access to literature & 14 & $\begin{array}{l}\text { (i) Availability and access to recent literature } \\
\text { (ii) Need broader access to online journals and databases }\end{array}$ \\
\hline Resources & 26 & $\begin{array}{l}\text { (i) Getting access research articles } \\
\text { (ii) Availability of resources }\end{array}$ \\
\hline Staffing and teamwork & 15 & $\begin{array}{l}\text { (i) Require adequate staffing } \\
\text { (ii) Develop clinical leaders with research skills to serve as role models }\end{array}$ \\
\hline $\begin{array}{l}\text { Awareness and creating a } \\
\text { research culture }\end{array}$ & 21 & $\begin{array}{l}\text { (i) "The public needs to be aware of what EBP is, otherwise they will demand to continue } \\
\text { unnecessary investigations" }\end{array}$ \\
\hline Total & 219 & \\
\hline
\end{tabular}

(70.2\%), "administration will not allow implementation" (69.2\%), "physicians will not cooperate with implementation" $(68.7 \%)$, and "nurse does not have time to read research" $(65.2 \%)$. For the individual factors, which are considered nurse-related barriers, "nurse does not feel capable of evaluating the quality of the research" (67.7\%) was reported as the highest barrier. More than half of the respondents considered "the nurse sees little benefit for self" $(6.2 \%)$ and "the nurse is unwilling to change/try new ideas" (60.6\%) as barriers. Likewise, about half of the respondents did not consider "the nurse is isolated from knowledgeable colleagues with whom to discuss the research" (52.5\%) a barrier. Communication factors measured the nurses' perceptions of the presentation and accessibility of the research. The respondents reported that difficulty in understanding the statistical analysis (83.3\%) and relevant literature not being compiled in one location $(80.3 \%)$ were the biggest barriers. In addition, $56.6 \%$ reported that research reports are not readily available. The quality of research, which is known as the characteristics of the innovation, measured the nurses' perceptions of quality of research. More than half of the respondents (56.6\%) felt that "the nurse is uncertain whether to believe the results of research" was not a barrier.

The main barriers to using research evidence in nursing practice among nurses working in MHSC were items appearing under organisational (mean 3.03) followed by the adopter (mean 2.54) factors. These findings are similar to previous studies in which lack of organisation support was cited as a barrier $[4,6,9,13,20]$. BARRIER Scale items in rank order by each factor scores are summarised in Table 2 .

6.2. Facilitators of Research Utilisation. The respondents identified numerous facilitators to research use by using the open-ended questions. A total of 219 facilitators were grouped into broad themes (see Table 3). Availability of time, support and encouragement from the management and administration, advancing nurses knowledge, providing training opportunities, availability of resources, access to literature, teamwork, and awareness and creating a research culture appeared to be the most common facilitators. The most frequently cited facilitator was support, encouragement, and recognition from the management and administration (76 suggestions). The second most frequently cited facilitator of EBP was increasing the availability of time (45 suggestions). A number of nurses expressed that they need time to discuss ideas with colleagues, develop protocols, read research articles, and implement new ideas. The third most frequently suggested facilitator was related to nurses' knowledge (22 suggestions). Improvement of in-service classes-including discussion of research papers during the in-service programs and introducing regular professional 
development-was suggested by many respondents. Some participants also suggested providing opportunities to attend conferences internationally (see Table 3).

\section{Discussion}

The self-reporting from the questionnaire by the nurses shows that a lot of work is still needed if EBP is to be implemented in the Maldives. More than 30\% of respondents reported that they hardly ever sought research evidence for their clinical practice. Some of the reasons given for not using research were the same as in many other studies [2, 11, 13]. Additionally, it was found that more than half of the respondents were not familiar with the term EBP. It is evident that there are many factors for this. Some of these-such as literature not being compiled in one place, insufficient time on the job to implement new ideas, and lack of support from administration and physicians-are problems that are common in many healthcare organisations.

In this present study, major barriers to research use were found to be most often related to organisational factors. Insufficient time was reported as a major barrier; hence, consideration needs to be given to ways of improving nurses' time management. This finding is supported by several other studies $[10,12,21,22]$. An explanation for insufficient time on the job could be that most nursing practices are more tradition based than evidence based, which might result in the increased workload. However, it could also be due to poor time management. Additionally, a lack of trained clinicians could lead to increased workload and poor time management.

In an Australian study, 149 nurses indicated that three main barriers to research utilization were insufficient time on the job to implement new ideas, not enough time to read research, and nurses being unaware of research [10]. In summary, that study identified that six of the top 10 barriers to implementing research findings were related to the organisation, as reflected by the responses to the items: "insufficient time on the job," "administration will not allow implementations," "physicians will not cooperate," and "nurses do not have time to read research."

In this study, these perceived that reported barriers are consistent with the literature [10]. There are many different ways of increasing the knowledge of nurses, including introducing in-service classes and conducting professional development sessions during the overlapping time between shifts, instead of asking nurses to attend during their days off, and strengthening professional development by providing incentives to undertake at least 20 hours of professional development every year. Providing a project day for clinical nurses whose major role is updating the clinical guidelines and procedures may ultimately increase interest in developing evidence based care. However, there is no right or wrong approach to do this. Given that some of the clinical nursing practices in the Maldives are based on tradition and habit, creative thinking may be needed to help bridge the gap between actual current practice and expected best nursing practice.
Support, encouragement, and recognition from the management and administration were the most frequent facilitators for research utilisation. A reward system may be needed to help profile innovative practice development achievements in the Maldives healthcare to support and encourage other novice evidence based practitioners. Nurses' research values, skills, and awareness are important factors that need to be considered. Educators can help nurses develop a positive selfimage by providing them with theory and practical experience that encourages more independent function and allows them a professional role to provide unique and essential services to the community.

\section{Limitations of Study}

This study was conducted using convenience sampling of participants using a self-reporting questionnaire. Hence, a limitation of this study would be the generalizability of the research findings due to sampling techniques and sample size. However, it is believed that the findings may be relevant to nurses in similar organisations in other developing countries. Another limitation inherent in this study derived from the number of respondents who selected "no opinion" or failed to respond, which resulted in low internal consistency of the BARRIER Scale. However, the results are consistent with previously conducted studies. Nevertheless, it had produced information about the perceived barriers in the Maldives that could be used while implementing EBP in the country.

\section{Implications for Administrators}

The findings from this study show that administrators could support the development of EBP by allowing nurses time and resources to enhance their skills related to EBP. Hence, strategies need to be implemented to improve knowledge about EBP. Nursing administrators can support nurses by providing time for activities that promote EBP development. Many approaches can be taken to address this issue, such as introducing in-service classes and conducting professional development sessions during the overlapping time between shifts, instead of asking nurses to attend during their days off, and strengthening professional development by providing incentives to undertake at least 20 hours of professional development every year. In addition, providing a project day to clinical nurses whose major role is updating the clinical guidelines and procedures may ultimately increase interest in developing evidence based care. However, if sustained change is to be achieved, then national policies that have specific implications for EBP in the country are urgently required.

\section{Conclusion}

The findings from this study confirm that EBP is a new concept in the Maldives. This research was the first study undertaken to examine the barriers to using research evidence in nursing practice among nurses in the Maldives. This research aimed to identify the barriers and facilitators in five healthcare facilities in the Maldives. The results indicated that 
there are a range of barriers of concern to nurses, and these are consistent with results that have been reported in previous research internationally. To enhance EBP, strategies should be placed to minimise barriers and enhance the facilitators of research utilisation. However, the findings from this study also indicate that the majority of the nurses are not familiar with EBP, highlighting the need for professional nursing development in the country.

\section{Conflict of Interests}

The authors declare that there is no conflict of interests regarding the publication of this paper.

\section{Acknowledgments}

One of the authors would like to thank Malé Health Service Corporation for giving permission to conduct this study and would also like to thank Professor Sandra G Funk for permission to use the BARRIER Scale. One of the authors wishes to acknowledge and thank all the nurses who completed the questionnaire.

\section{References}

[1] D. L. Sackett, W. M. C. Rosenberg, J. A. M. Gray, R. B. Haynes, and W. S. Richardson, "Evidence based medicine: What it is and what it isn't. It's about integrating individual clinical expertise and the best external evidence," British Medical Journal, vol. 312, no. 7023, pp. 71-72, 1996.

[2] J. S. Ashley, Barriers and facilitators to research utilization as perceived by critical care nurses [Ph.D. thesis], University of California, San Francisco, Calif, USA, 2005.

[3] A.-M. Boström, K. N. Kajermo, G. Nordström, and L. Wallin, "Barriers to research utilization and research use among registered nurses working in the care of older people: does the BARRIERS Scale discriminate between research users and nonresearch users on perceptions of barriers?" Implementation Science, vol. 3, no. 1, article 24, 2008.

[4] R. M. Bryar, S. J. Closs, G. Baum et al., "The Yorkshire BARRIERS project: diagnostic analysis of barriers to research utilisation," International Journal of Nursing Studies, vol. 40, no. 1, pp. 73-84, 2003.

[5] S. J. Closs, G. Baum, R. M. Bryar, J. Griffiths, and S. Knight, "Barriers to research implementation in two Yorkshire hospitals," Clinical Effectiveness in Nursing, vol. 4, no. 1, pp. 3-10, 2000.

[6] S. G. Funk, M. T. Champagne, R. A. Wiese, and E. M. Tornquist, "Barriers: the barriers to research utilization scale," Applied Nursing Research, vol. 4, no. 1, pp. 39-45, 1991.

[7] L. McCleary and G. T. Brown, "Barriers to paediatric nurses' research utilization," Journal of Advanced Nursing, vol. 42, no. 4, pp. 364-372, 2003.

[8] T. Moreno-Casbas, C. Fuentelsaz-Gallego, Á. G. de Miguel, E. González-María, and S. P. Clarke, "Spanish nurses' attitudes towards research and perceived barriers and facilitators of research utilisation: a comparative survey of nurses with and without experience as principal investigators," Journal of Clinical Nursing, vol. 20, no. 13-14, pp. 1936-1947, 2011.
[9] K. Parahoo, "Barriers to, and facilitators of, research utilization among nurses in Northern Ireland," Journal of Advanced Nursing, vol. 31, no. 1, pp. 89-98, 2000.

[10] A. Retsas and M. Nolan, "Barriers to nurses' use of research: an Australian hospital study," International Journal of Nursing Studies, vol. 36, no. 4, pp. 335-343, 1999.

[11] D. R. Thompson, J. P. C. Chau, and V. Lopez, "Barriers to, and facilitators of, research utilisation: a survey of Hong Kong registered nurses," International Journal of Evidence-Based Healthcare, vol. 4, no. 2, pp. 77-82, 2006.

[12] S. G. Funk, E. M. Tornquist, and M. T. Champagne, "Barriers and facilitators of research utilization. An integrative review," Nursing Clinics of North America, vol. 30, no. 3, pp. 395-407, 1995.

[13] A. M. Hutchinson and L. Johnston, "Bridging the divide: a survey of nurses' opinions regarding barriers to, and facilitators of, research utilization in the practice setting," Journal of Clinical Nursing, vol. 13, no. 3, pp. 304-315, 2004.

[14] S. L. Pagoto, B. Spring, E. J. Coups, S. Mulvaney, M. F. Coutu, and G. Ozakinci, "Barriers and facilitators of evidence-based practice perceived by behavioral science realth professionals," Journal of Clinical Psychology, vol. 63, no. 7, pp. 695-705, 2007.

[15] E. Patiraki, C. Karlou, D. Papadopoulou et al., "Barriers in implementing research findings in cancer care: the Greek registered nurses perceptions," European Journal of Oncology Nursing, vol. 8, no. 3, pp. 245-256, 2004.

[16] K. N. Kajermo, G. Nordström, Å. Krusebrant, and H. Björvell, "Barriers to and facilitators of research utilization, as perceived by a group of registered nurses in Sweden," Journal of Advanced Nursing, vol. 27, no. 4, pp. 798-807, 1998.

[17] G. W. Marsh, M. Nolan, and S. Hopkins, "Testing the revised barriers to research utilization scale for use in the UK," Clinical Effectiveness in Nursing, vol. 5, no. 2, pp. 66-72, 2001.

[18] E. M. Rogers, Diffusion of Innovations, Free Press, New York, NY, USA, 5th edition, 2003.

[19] D. Polit-O'Hara, Data Analysis \& Statistics for Nursing Research, Appleton \& Lange, Stamford, Colo, USA, 1996.

[20] A. L. Dans and L. F. Dans, "Introduction to EBP in developing countries," September 2013, http://www.cebm.utoronto.ca/syllabi/devl/intro.htm.

[21] C. Hicks, "Barriers to evidence-based care in nursing: historical legacies and conflicting cultures," Health Services Management Research, vol. 11, no. 3, pp. 137-147, 1998.

[22] A. McColl, H. Smith, P. White, and J. Field, "General practitioners' perceptions of the route to evidence based medicine: a questionnaire survey," British Medical Journal, vol. 316, no. 7128, pp. 361-365, 1998. 


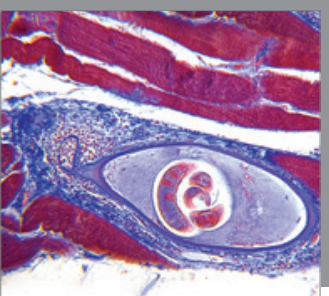

Gastroenterology

Research and Practice
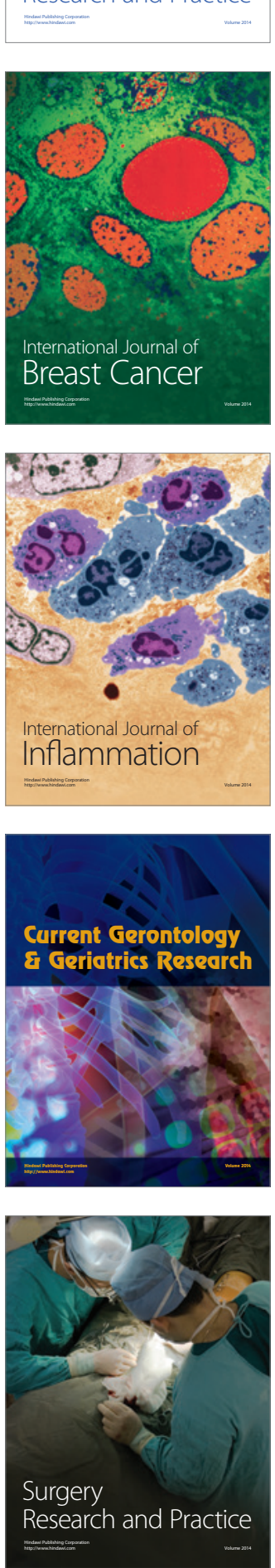

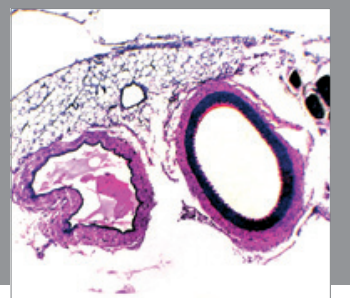

International Journal of Hypertension
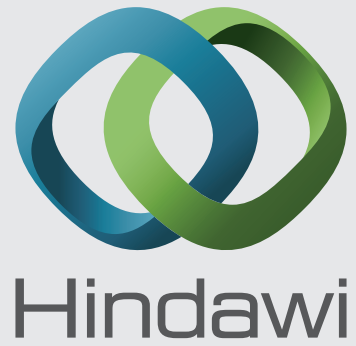

Submit your manuscripts at http://www.hindawi.com
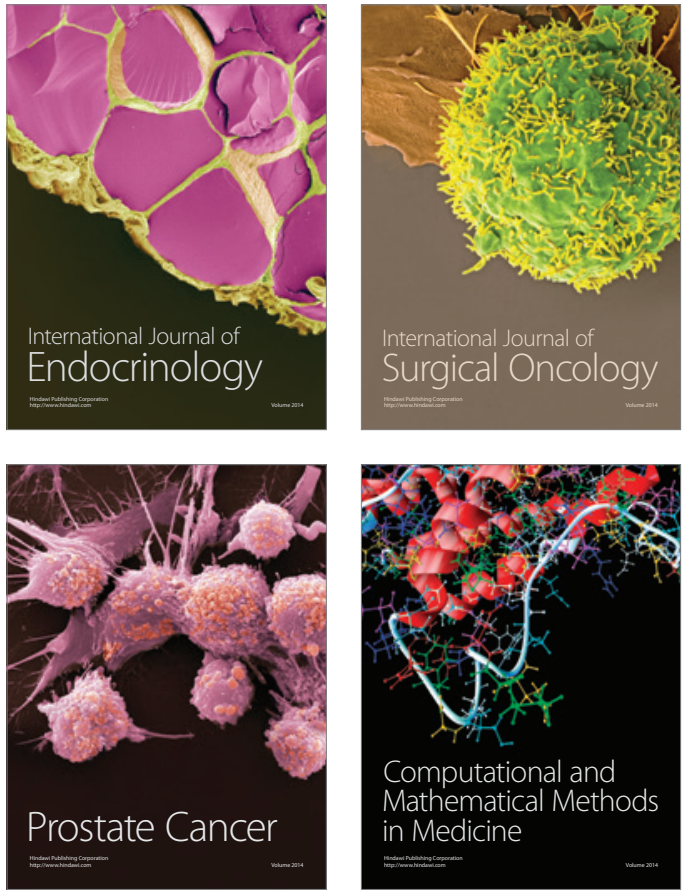
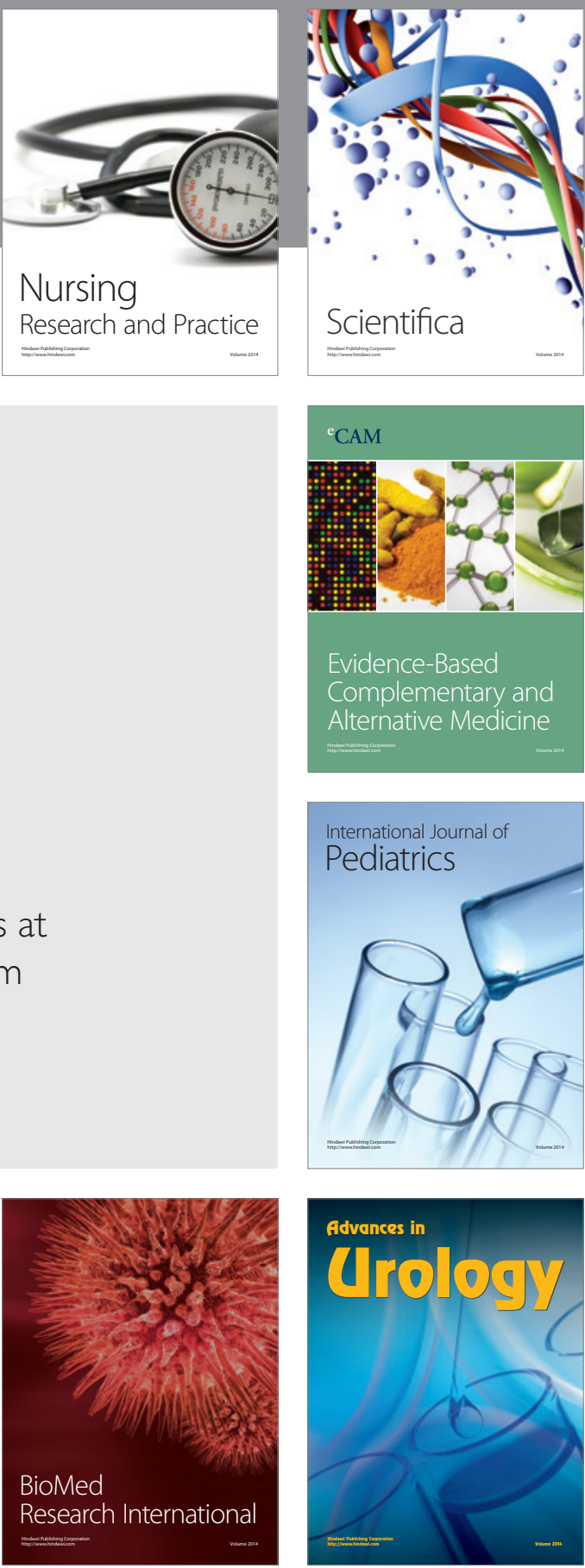

Nursing

Research and Practice

Scientifica

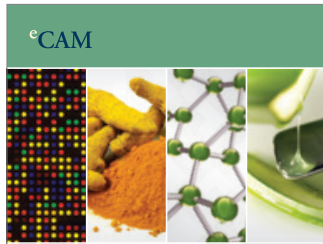

Evidence-Based

Complementary and Alternative Medicine
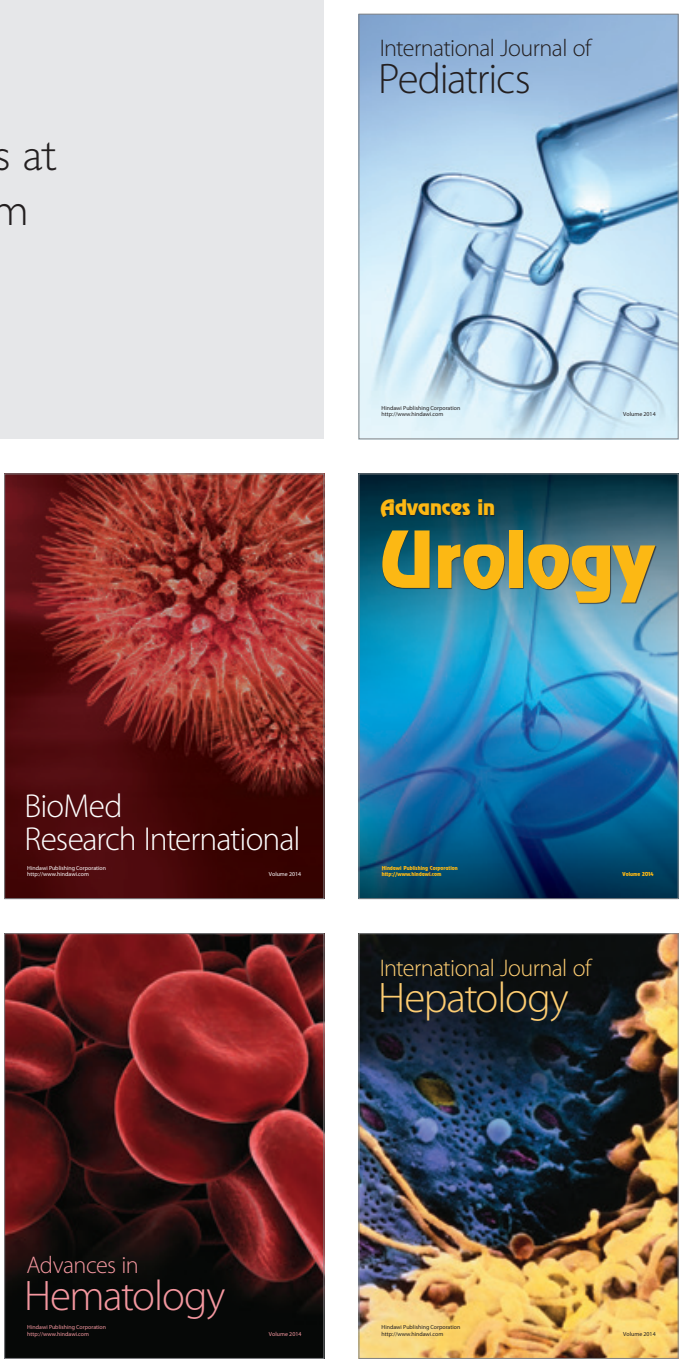